\title{
Lista das espécies de Pantophthalmidae e Stratiomyidae (Diptera, Stratiomyioidea) do estado do Mato Grosso do Sul, Brasil
}

\author{
Diego Aguilar Fachin', Cecília Kosmann², Cristiane V. A. Pujol-Luz ${ }^{3}$ \& José Roberto Pujol-Luz ${ }^{2}$
}

\author{
1. Universidade de São Paulo, Faculdade de Filosofia, Ciências e Letras de Ribeirão Preto, Departamento de Biologia, Av. Bandeirantes $3900,14040-901$ \\ Ribeirão Preto, SP, Brasil. \\ 2. Universidade de Brasília, Instituto de Ciências Biológicas, Departamento de Zoologia, 70910-900 Brasília, DF, Brasil. (jrpujol@unb.br) \\ 3. Universidade Católica de Brasília, Laboratório de Zoologia, QS 7 lote 1, BI. M/ s. 331, 72030-170 Taguatinga, DF, Brasil.
}

Recebido 8 dezembro 2016

Aceito 6 fevereiro 2017

DOI: $10.1590 / 1678-4766 e 2017130$

\begin{abstract}
Checklist of species of Pantophthalmidae and Stratiomyidae (Diptera, Stratiomyioidea) of the state of Mato Grosso do Sul, Brazil. In this paper we provided a checklist of the Pantophthalmidae and Stratiomyidae species registered in the state of Mato Grosso do Sul, Brazil. Only one species of Pantophthamildae is recorded for the state. Stratiomyidae is represented by 12 species in nine genera: Acrochaeta Wiedemann (1), Artemita Walker (1), Neochauna Williston (1), Chrysochlorina James (3), Cyphomyia Wiedemann (1), Hermetia Latreille (2), Ptecticus Loew (1), Sargus Fabricius (1), and Hoplitimyia James (1).
\end{abstract}

KEYWORDS. Central-Western region, distribution, diversity, taxonomy, Biota-MS Program.

RESUMO. Nesse artigo é fornecida uma lista de espécies de Pantophthalmidae e Stratiomyidae registradas no estado Mato Grosso do Sul. Apenas uma espécie de Pantophthalmidae possui registro para o estado. Stratiomyidae é representada por 12 espécies distribuídas em nove gêneros: Acrochaeta Wiedemann (1), Artemita Walker (1), Neochauna Williston (1), Chrysochlorina James (3), Cyphomyia Wiedemann (1), Hermetia Latreille (2), Ptecticus Loew (1), Sargus Fabricius (1), and Hoplitimyia James (1).

PALAVRAS-CHAVE. Centro-Oeste, diversidade, distribuição, taxonomia, Programa Biota-MS

Pantophthalmidae é uma família de moscas grandes e robustas, pertencente à subordem Brachycera, superfamília Stratiomyoidea (PAPE et al., 2011), como grupo-irmão do clado Stratiomyidae + Xylomyidae (WoodLEy et al., 2009). São popularmente conhecidos como moscas-damadeira, sendo que os adultos, em sua maioria, apresentam coloração castanho-escura a avermelhada ou preta, com listras longitudinais no mesonoto. São distintos dos outros braquíceros basais por apresentarem grandes empódios pulviliformes, ausência de esporões nas tíbias anteriores e posteriores, e presença de um distinto tufo de cerdas abaixo ou próximo ao espiráculo posterior (WoODLEY, 2009a). As asas são hialinas, com manchas de padrões variados, amarelas ou marrons. Os olhos são grandes e ocupam a maior parte da cabeça. As fêmeas são dicópticas e os machos holópticos, ambos com oito segmentos na antena. As larvas possuem corpo com 12 segmentos, o primeiro segmento do tórax e o último do abdômen são fortemente quitinizados. As larvas são anfipnêusticas, com um par de espiráculos torácicos e um par de espiráculos abdominais; exceto na larva de primeiro instar, que possui órgãos digitiformes ou flabeliformes na parte ventral do abdômen. Suas larvas atacam e perfuram árvores vivas ou mortas, formando galerias onde se alimentam provavelmente da matéria orgânica acumulada ou da sua fermentação (ANDRADE, 1930; GREENE \& URICH, 1931; THORPE, 1934). Aspectos da biologia do grupo foram tratados por VAL $(1976,1992)$.

A família é exclusiva da Região Neotropical, com 20 espécies descritas em dois gêneros: Pantophthalmus Thunberg (19 espécies) e Opetiops Enderlein (1 espécie) (PApaVero, 2009a; Val, 1976; Woodley, 2009a). No Brasil ocorrem dez espécies de Pantophthalmus, além da única espécie de Opetiops (PAPAVERo, 2009a; VAL, 1976). VAL (1976) fez uma revisão completa da família estudando a taxonomia e a evolução do grupo em diversos aspectos. Em Papavero (2009b), Val (1976) e Woodley (2009a) é possível encontrar chaves para os gêneros e em VAL (1976), chave para as espécies.

Stratiomyidae é uma família de moscas de tamanho médio ( 2 a $28 \mathrm{~mm}$ ) (com exceção dos Chiromyzinae, onde algumas fêmeas podem chegar a medir $34 \mathrm{~mm}$ ) que apresentam coloração e formato do corpo muito variado. Assim como Pantophthalmidae, são braquíceros da superfamília Stratiomyoidea (PAPE et al., 2011), como grupoirmão de Xylomyidae (WoODLEY, 1989; 2001; WoODLEY et al., 2009). Os adultos são facilmente reconhecidos pela venação das asas: veias radiais concentradas na parte anterior da asa e uma pequena célula discal da qual se irradiam 
as nervuras medianas; as larvas, assim como as larvas de Xylomyidae, apresentam a cutícula impregnada por carbonato de cálcio (Woodley, 1989; 2001; 2009b). As larvas podem ser aquáticas ou terrestres e frequentemente estão associadas à matéria orgânica em decomposição. De modo geral, tanto a morfologia quanto a biologia das formas imaturas das espécies brasileiras estão sendo estudadas (PuJOL-Luz \& Leite, 2001; Pujol-Luz \& Xerez, 1999; Xerez \& PujolLuZ, 2001; Xerez et al., 2002, 2003a,b, 2004; VianA \& Xerez, 2002; Viana et al., 2003; Pujol-Luz et al., 2004). Alguns outros aspectos de biologia (e.g., fonte de recursos) das espécies neotropicais também foram estudados (FonTENELLE et al., 2012).

É a família mais diversa e numerosa em termos de números de gêneros e espécies em Stratiomyoidea, com 2.865 espécies descritas em 380 gêneros para o mundo, sendo que 987 de 163 gêneros são conhecidas para a Região Neotropical (Woodley, 2001; 2011). Desse total, cerca de 330 espécies ocorrem no Brasil (JRPL, dados inéditos).

De acordo com o catálogo mundial (WoodLey, 2001), a família é dividida em 12 subfamílias: Antissinae, Beridinae, Chiromyzinae, Chrysochlorininae, Clitellarinae, Hermetiinae, Nemotelinae, Pachygastrinae, Parhadrestiinae, Raphiocerinae, Sarginae e Stratiomyinae. Dentre essas, Sarginae é a mais abundante na Região Neotropical, com 267 espécies (WoodLey, 2001; 2011). Uma parte da fauna Neotropical foi incansavelmente estudada por JAMES (1941, 1973a,b, 1974, 1975) e por JAMES \& MCFADDEN (1971; 1979; 1982), inclusive com chaves para subfamílias, gêneros e espécies, bem como um catálogo para a fauna. Ademais, chaves e sinopses para os gêneros da América Central podem ser encontrados em WoOdLEy (2009b).

\section{MATERIAL E MÉTODOS}

Foi realizada uma revisão na bibliografia taxonômica de todas as famílias de Stratiomyoidea (i.e. Pantophthalmidae, Stratiomyidae e Xylomyidae) para levantar as espécies conhecidas para o estado do Mato Grosso do Sul.

Pantophthalmidae: a lista foi elaborada com base nos registros do catálogo neotropical (PAPAVERO, 2009a). Bibliografia adicional foi consultada: RAPP \& SNOW (1945); Carrera \& D’Andretta (1957); Papavero (1967; 2002); Abreu \& Rocha (2003); Amat (2005).

Stratiomyidae: a lista de espécies foi realizada com base principalmente nos registros dos catálogos para a Região Neotropical (JAMES, 1973a) e mundial (WoODLEY, 2001; 2011). Anotações pessoais (JRPL, dados inéditos), bibliografia adicional (WiLliston, 1888; IIDE, 1963a,b, 1966; IIDE \& MiLETI, 1976; JAMES \& MCFADDEN, 1982; LeAL, 1979; Ururahy-Rodrigues, 2004; Godoy, 2006), além de material do Museu de Zoologia da Universidade de São Paulo (MZUSP).

Xylomyidae: essa família não é abordada aqui, pois não há registros de espécies para o estado do Mato Grosso do Sul.

\section{RESULTADOS E DISCUSSĀO}

\section{Lista das espécies de Pantophthalmidae e Stratiomyidae do Mato Grosso do Sul}

\section{PANTOPHTHALMIDAE}

\section{Pantophthalmus Thunberg, 1819}

kerteszianus Enderlein, 1914. Localidade-tipo: Peru, Mariscal Cáceres, Juanjui. Distribuição: Bolívia, Brasil (Amazonas, Pará, Mato Grosso do Sul), Colômbia (Cochabamba, Santa Cruz), Panamá, Peru. Ref.: PAPAVERo (2009a).

\section{STRATIOMYIDAE CHRYSOCHLORININAE}

\section{Chrysochlorina James, 1939}

albipes James, 1939. Localidade-tipo: Brasil, Goiás. Distribuição: Brasil (Espírito Santo, Goiás, Mato Grosso do Sul, Minas Gerais, Rio de Janeiro, São Paulo, Paraná, Santa Catarina). Ref.: IIDE (1966); JAMEs (1973a); WoodLEY (2001).

incompleta Curran, 1929. Localidade-tipo: Brasil, Rio de Janeiro. Distribuição: Bolívia, Brasil (Pará, Espírito Santo, Goiás, Mato Grosso do Sul, Rio de Janeiro, São Paulo). Ref.: IIDE (1966); JAMEs (1973a); WoOdLEy (2001). vespertilio (Fabricius, 1805). Localidade-tipo: “America meridional". Distribuição: México, Peru, Bolívia, Brasil (Goiás, Mato Grosso, Mato Grosso do Sul, São Paulo, Santa Catarina). Ref.: IIDE (1966); JAMEs (1973a); WoodLEY (2001).

\section{CLITELLARINAE}

\section{Cyphomyia Wiedemann, 1819}

leucocephala Wiedemann, 1819. Localidade-tipo: Brasil. Distribuição: Argentina, Brasil (Mato Grosso do Sul, Rio de Janeiro e Paraná), Equador, Paraguai, Peru. Ref.: IIDE (1963a); Woodley (2001).

\section{HERMETIINAE}

\section{Hermetia Latreille, 1804}

albitarsis Fabricius, 1805. Localidade-tipo: “América do Sul'. Distribuição: México (Tabasco, Yucatán), Costa Rica, El Salvador, Guatemala, Honduras, Panamá, Trinidad e Tobago, Venezuela, Equador, Peru, Guiana, Bolívia, Brasil (Mato Grosso do Sul, Santa Catarina), Paraguai.

illucens (Linnaeus, 1758). Localidade-tipo: “América do Sul". Distribuição: México (Chiapas, Chihuahua, Jalisco, Nuevo León, Oaxaca, Quintana Roo, San Luis Potosí, Sinaloa, Tamaulipas, Veracruz, Yucatán), Panamá, República Dominicana, El Salvador, Costa Rica, Belize, Porto Rico, Granada, Guatemala, Haiti, Honduras, Jamaica, Ilhas Virgens, Trinidad e Tobago, Colômbia, Equador, Chile, Argentina, 
Brasil (Mato Grosso, Mato Grosso do Sul), Dominica, Guiana, Paraguai, Peru, Suriname, Venezuela, Uruguai. Ref.: IIDE \& Mileti (1976).

\section{PACHYGASTRINAE}

\section{Artemita Walker, 1854}

podexargenteus Enderlein, 1914. Localidade-tipo: Brasil, Santa Catarina. Distribuição: Brasil (Santa Catarina, Mato Grosso do Sul), Paraguai. Ref.: UrURAHY-Rodrigues (2004); WoodLEy (2001).

\section{Neochauna Williston, 1896}

variabilis Loew, 1847. Localidade-tipo: Cuba. Distribuição: Brasil (Mato Grosso do Sul), Costa Rica, Cuba, El Salvador, México (Chiapas), Panamá, Peru. Ref.: Godoy (2006); WoOdLey (2001).

\section{SARGINAE}

\section{Acrochaeta Wiedemann, 1830}

fasciata Wiedemann, 1830. Localidade-tipo: Brasil. Distribuição: Brasil (Mato Grosso do Sul, Maracajú).

\section{Ptecticus Loew, 1855}

testaceus Fabricius, 1794:338. Localidade-tipo: “America meridionali”. Distribuição: México (Campeche, Chiapas, Colima, Michoacán, Oaxaca, Quintana Roo, San Luis Potosí, Tabasco, Tamaulipas, Veracruz, Yucatán), Panamá, Costa Rica, Honduras, Trindade Belize, Brasil (Amapá, Amazonas, Pará, Pernambuco, Bahia, Mato Grosso, Mato Grosso do Sul, Espírito Santo, Minas Gerais, Rio de Janeiro, São Paulo, Paraná, Santa Catarina), Argentina. Ref.: LeAl \& Oliveira (1979); JAMES \& MCFADDEN (1982); Pujol-Luz \& Leite (2001); Woodley (2001).

\section{Sargus Fabricius, 1798}

fasciatus Fabricius, 1805. Localidade-tipo: “America meridionali”. Distribuição: México (Campeche, Colima, Guerrero, Jalisco, México, Morelos, Nayarit, Nuevo León, Oaxaca, San Luis Potosí, Tamaulipas, Veracruz, Yucatán), Panamá, Porto Rico, República Dominicana, Bahamas, Costa Rica, Cuba, Granada, Guatemala, Honduras, Jamaica, Dominica, El Salvador, Equador, Brasil (Mato Grosso, Mato Grosso do Sul). Ref.: Williston (1888); JAMES (1982); WoOdLey (2001); FACHIN (2011).

\section{STRATIOMYINAE}

\section{Hoplitimyia James, 1934}

mutabilis (Fabricius), 1787:331. Localidade-tipo: Guiana Francesa. Distribuição: Argentina, Brasil (Mato Grosso do Sul), Costa Rica, El Salvador, Guiana Francesa, Honduras, México (Baja California, Chiapas, Colima, Durango, Guanajuato, Guerrero, Jalisco, Michoacán,
Morelos, Nayarit, Nuevo León, Oaxaca, Puebla, San Luis Potosí, Sinaloa, Sonora, Tamaulipas, Veracruz), Nicarágua.

Para o estado do Mato Grosso do Sul, apenas uma espécie de Pantophthalmidae foi apontada: Pantophthalmus kerteszianus Enderlein, 1914. Apesar dessa inexpressiva representatividade da família no estado, é provável que algumas das outras dez espécies reconhecidas para o Brasil ocorram no Mato Grosso do Sul. Há registros de P. kerteszianus danificando espécies de Euphorbiaceae: Croton matourensis (ABREU \& RochA, 2003) e de Fabaceae: Schizolobium parahyba (Vell.) S.F. Blake var. amazonicum (Huber ex Ducke) Barneby (Lunz et al., 2010) no Brasil. Outras espécies de pantofitalmídeos danificam uma variedade de espécies de plantas amplamente distribuídas no Brasil. Dentre elas destacamos as Araucariaceae, Bombacaceae, Lauraceae, Moraceae e Casuarinaceae (VAL, 1976). Com certeza uma investigação mais adequada considerando os hábitos e a biologia da mosca-da-madeira ampliará o número de espécies para o estado do Mato Grosso do Sul.

Considerando Stratiomyidae, foram registradas apenas doze espécies distribuídas em nove gêneros e seis subfamílias: Chrysochlorina albipes, C. incompleta, $C$. vespertilio (Chrysochlorininae); Cyphomyia leucocephala (Clitellarinae); Hermetia albitarsis; Hermetia illucens (Hermetiinae); Artemita podexargenteus, Neochauna variabilis (Pachygastrinae); Acrochaeta fasciata, Ptecticus testaceus, Sargus fasciatus (Sarginae); Hoplitimyia mutabilis (Stratiomyinae). A riqueza total da família no estado é baixíssima e pouco estudada. Provavelmente isto é um reflexo das poucas coletas feitas no local e também da falta de conhecimento com relação à amplitude da distribuição geográfica das espécies. Para fins de comparação, o estado do Mato Grosso - também da região Centro-Oeste do país apresenta um número muito superior ao encontrado no Mato Grosso do Sul: 31 espécies em 20 gêneros e sete subfamílias (Williston, 1888; James, 1973a; Leal, 1979; Pujol-Luz, 2000; Pujol-Luz \& Leite, 2001; Pimentel \& Pujol-Luz, 2001; Woodley, 2001; Godoy, 2006; FACHIN, 2011). Essa diferença não deve servir de parâmetro para definir maior diversidade em um do que no outro, mas apenas explicita o pouco conhecimento com relação às espécies, pois é provável que pelo menos uma parte das que ocorrem no estado vizinho também podem ser encontradas no Mato Grosso do Sul .

Em termos de representatividade e quantidade de exemplares, a coleção mais significativa de Pantophthalmidae e Stratiomyidae é a do Museu de Zoologia da Universidade de São Paulo. Entretanto, outras coleções do País também merecem destaque, pois incluem importantes espécimes de referência, como as coleções do Instituto Nacional de Pesquisas da Amazônia (INPA), Manaus, AM; Museu Nacional da Universidade Federal do Rio de Janeiro (MNRJ), Rio de Janeiro, RJ; Museu Paraense Emilio Goeldi (MPEG), Belém, PA. No exterior, destacam-se as coleções do American Museum of Natural History, Nova Iorque, EUA; Canadian National Collection, Ottawa Canadá; National Museum of Natural History, Washington D.C., EUA e Natural History Museum, Londres, Inglaterra. 
Atualmente, os principais grupos de pesquisa no Brasil dedicados ao estudo destes dípteros estão localizados nos estados de São Paulo (Faculdade de Filosofia, Ciências e Letras de Ribeirão Preto, Universidade de São Paulo), Rio de Janeiro (Universidade Federal Rural do Rio de Janeiro) e no Distrito Federal (Universidade de Brasília) e desenvolvem pesquisas sobre biologia, taxonomia, sistemática, biogeografia e desenvolvimento pós-embrionário.

Agradecimentos. Os autores agradecem a Fundação de Apoio ao Desenvolvimento do Ensino, Ciências e Tecnologia do Estado de Mato Grosso do Sul (Fundect) e a Superintendência de Ciências e Tecnologia do Estado de Mato Grosso do Sul (Sucitec/MS) pelo convite de participação neste fascículo especial da Iheringia, série Zoologia e o suporte financeiro para sua publicação. As seguintes agências de fomento à pesquisa: Conselho Nacional de Desenvolvimento Científico e Tecnológico (CNPq); Fundação de Amparo à Pesquisa do Estado de São Paulo (FAPESP); Fundação de Apoio à Pesquisa do Distrito Federal (FAPDF); Coordenação de Aperfeiçoamento de Pessoal de Nível Superior (CAPES); Financiadora de Estudos e Projetos (FINEP), Pró-Reitoria de Pesquisa e Pós-Graduação e Pesquisa da Universidade Católica de Brasília (UCB). Este trabalho recebeu apoio financeiro do Conselho Nacional de Desenvolvimento Científico e Tecnológico (CNPq, processo SISBIOTA 563256/2010-9) e da Fundação de Amparo à Pesquisa do Estado de São Paulo (FAPESP), processos: DAF (2011/14472-6), SISBIOTA (2010/52314-0).

\section{REFERÊNCIAS BIBLIOGRÁFICAS}

Abreu, R. L. S. \& Rocha, R. A. 2003. Ocorrência de Pantophthalmus kerteszianus Enderlein (Diptera: Brachycera) em Croton lanjowvensis [sic] (Euphorbiaceae) em Manaus, Estado do Amazonas. Neotropical Entomology 32(2):361-362.

Амат, E. C. 2005. New records of timber flies (Diptera: Pantophthalmidae) from Colombia. Entomotropica 20(2):95-96.

Andrade, E. N. De. 1930. Bibliographia da "mosca da madeira". Chacáras e Quintais 41(5):536.

Carrera, M. \& D'AndretTa, M. A. V. 1957. Sôbre a família Pantophtalmidae (Díptera). Arquivos de Zoologia do Estado de São Paulo 10:253-330.

FACHIN, D. A. 2011. Diversidade e Distribuição de Sarginae (Diptera: Stratiomyidae) na Floresta Atlântica. Monografia de conclusão de curso. Ribeirão Preto, Universidade de São Paulo.

Fontenelle, J. C. R.; Viana-Silva, F. E. C. \& Martins, R. P. 2012. Use of Plant Resources by Merosargus (Diptera, Stratiomyidae, Sarginae) larvae. Psyche 2012:1-10.

Godoy, F. S. P. 2006. Morfologia da espermateca e forquilha genital de algumas espécies das subfamílias neotropicais de Stratiomyidae (Insecta, Diptera). Dissertação de Mestrado. Manaus, Universidade Federal do Amazonas.

Greene, C. T. \& Urich, F. W. 1931.The immature stages of Pantophthalmus tabaninus Thunberg, with biological notes. Transactions of the Entomological Society of London 79:277-282.

IIDE, P. 1963a. Contribuição ao conhecimento do gênero Cyphomyia Wiedemann 1819 (Diptera, Stratiomyidae). Memórias do Instituto Oswaldo Cruz 61(1):25-39.

IIDE, P. 1963b. Contribuição ao conhecimento do gênero Chordonota Gerstaecker, 1857 (Diptera, Stratiomyidae). Memórias do Instituto Oswaldo Cruz 61(3):401-409.

IIDE, P. 1966. Estudo sôbre as espécies brasileiras do gênero Chrysochlorina James, 1939 (Diptera, Stratiomyidae). Arquivos de Zoologia 14(2):69112.

Iide, P. \& Mileti, D. I. C. 1976. Estudos morfológicos sobre Hermetia illucens (Linnaeus, 1758) (Diptera, Stratiomyidae). Revista Brasileira de Biologia 36(4):923-935.

James, M. T. 1941. A preliminary study of the New World Geosarginae (Diptera: Stratiomyidae). Lloydia 4:300-309.

James, M. T. 1973a. Family Stratiomyidae. In: PAPAVEro, N. ed. A catalogue of the Diptera of the Americas south of the United States. São Paulo, Departamento de Zoologia, Secretaria de Agricultura, p.1-95.
JAMES, M. T. 1973b. A preliminary review of the Stratiomyidae of Chile. Part I. Revista Chilena de Entomología 7:11-23.

James, M. T. 1974. The genus Nemotelus in South America (Diptera, Stratiomyidae). Melanderia 14:1-22.

JAMES, M. T. 1975. A preliminary review of the Stratiomyidae of Chile. Part II. Melanderia 20:1-28.

James, M. T. \& McFadden, M. W. 1971. The genus Merosargus in Middles America and the Andean Subregion (Diptera: Stratiomyidae). Melanderia 7:1-76.

JAMES, M. T. \& MCFADDEN, M. W. 1979. The Stratiomyinae (Diptera, Stratiomyidae) of Middle America. Melanderia 32:1-40.

James, M. T. \& McFadden, M. W. 1982. The Sarginae (Diptera: Stratiomyidae) of Middle America. Melanderia 40:1-50.

Leal, M. C. A\& Oliveira, M. H. C. C. 1979. Revisão das espécies brasileiras do gênero Ptecticus Loew, 1855 (Diptera: Stratiomyidae). Anais da Universidade Federal Rural de Pernambuco, Ciências Biológicas 4:37-102

Lunz, A. M.; Batista, T. F. C.; Rosário, V. do S. V.; Monteiro, O. M. \& Mahon, A. C. 2010. Ocorrência de Pantophthalmus kerteszianus e P. chuni (Diptera: Pantophthalmidae) em paricá, no Estado do Pará. Pesquisa Florestal Brasileira 30(61):71-74.

Papavero, N. 1967. Family Pantophthalmidae. In: Papavero, N. ed. A catalogue of the Diptera of the Americas south of the United States. São Paulo, Departamento de Zoologia, Secretaria de Agricultura, p.1-8.

Papavero, N. 2002. Insecta - Diptera - Pantophthalmidae. Fauna da Amazônia Brasileira 11:1-4.

Papavero, N. 2009a. Catalogue of Neotropical Diptera. Pantophthalmidae. Neotropical Diptera 19:1-11.

Papavero, N. 2009b. Manual of Neotropical Diptera. Pantophthalmidae. Neotropical Diptera 20:1-11.

Pape, T.; Blagoderov, V. \& Mostovski, M. B. 2011. Animal Biodiversity - Order Diptera. Zootaxa 3148:222-229.

Pimentel, T. \& Pujol-Luz, J. R. 2001. Os gêneros de Raphiocerinae (Diptera, Stratiomyidae) do Brasil e algumas espécies da América do Sul. Parte 2- A Tribo Raphiocerini (sensu Schiner). Contribuições Avulsas sobre a História Natural do Brasil, Série Zoologia 33:1-31.

Pujol-Luz, J. R. 2000. Panacris proxima Kertész, 1908 new synonym of Panacris lucida Gerstaecker, 1857 (Diptera, Stratiomyidae) with notes on the male terminalia. Studia Dipterologica 7:155-159.

Pujol-Luz, J. R. \& Leite, F. M. 2001. Descrição do último ínstar larval e do pupário de Ptecticus testaceus (Fabr.) (Diptera: Stratiomyidae). Neotropical Entomology 30(4):587-591.

Pujol-Luz, J. R. \& Xerez, R. DE. 1999. The larva of Chalcidomorphina aurata Enderlein 1914 (Diptera: Stratiomyidae) from "Ilha de Marambaia," Rio de Janeiro, Brasil. Proceedings of the Entomological Society of Washington 101(2):295-299.

Pujol-Luz, J. R.; Xerez, R. DE \& Vianna, G. G. 2004. Descrição do pupário de Raphiocera armata (Wiedemann) (Diptera, Stratiomyidae) da Ilha da Marambaia, Rio de Janeiro, Brasil. Revista Brasileira de Zoologia 21(4):995-999.

RaPP, F. \& SNOw, W. E. 1945. Catalogue of the Pantophthalmidae of the world [sic]. Revista de Entomologia 16:252-254.

ThORPE, W. H. 1934. Observations on the structure, biology and systematic position of Pantophthalmus tabaninus Thunb. (Diptera, Pantophthalmidae). Transactions of the Entomological Society of London 82:5-22.

UrURAhY-Rodrigues, A. 2004. Artemita bicolor Kertész, novo sinônimo de Artemita podexargenteus Enderlein, (Diptera, Stratiomyidae) com notas nas terminálias masculinas e femininas. Revista Brasileira de Zoologia 21(2):397-402.

VAL, F. C. Do. 1976. Systematics and evolution of the Pantophthalmidae (Diptera, Brachycera). Arquivos de Zoologia 27(2):51-164.

VAL, F. C. Do. 1992. Pantophthalmidae of Central America and Panama (Diptera). In: Quintero, D. \& Aiello, A. eds. Insects of Panama and Mesoamerica Selected Studies. New York, Oxford University Press, p.600-610.

Viana, G. G. \& Xerez, R. DE. 2002. Descrição do pupário de Sargus thoracicus Macquart (Diptera, Stratiomyidae, Sarginae). Revista Brasileira de Zoologia 19(Supl. 2):79-84.

Viana, G. G.; Pujol-Luz, J. R. \& Xerez, R. De. 2003. Descrição da Larva e do Pupário de Auloceromyia vespiformis Lindner (Diptera: Stratiomyidae, Clitellariinae). Neotropical Entomology 32(1):69-74. 
WiLliston, S. W. 1888. Diptera Brasiliana, ab H. H. Smith collecta. Part I-Stratiomyidae, Syrphidae. Transactions of the American Entomological Society 15:243-292.

Woodley, N. E. 1989. Phylogeny and classification of the "orthorrhaphous" Brachycera. In: McAlPINE J. F. \& Wood, D. M. eds. Manual of Nearctic Diptera Monograph. Ottawa, Agriculture Canada, p.13711395.

Woodley, N. E. 2001. A World Catalog of the Stratiomyidae (Insecta: Diptera). Myia 11:1-473.

Woodley, N. E. 2009a. Pantophthalmidae. In: Brown, B. V.; Borkent, A.; Cumming, J. M.; Wood, D. M.; Woodley, N. E. \& Zumbado, M. A. eds. Manual of Central American Diptera. Boca Raton, NCR Research Press, p.513-515.

Woodley, N. E. 2009b. Stratiomyidae. In: Brown, B. V.; Borkent, A.; Cumming, J. M.; Wood, D. M.; Woodley, N. E. \& Zumbado, M. A. eds. Manual of Central American Diptera. Boca Raton, NCR Research Press, p.521-549.

Woodley, N. E. 2011. A World Catalog of the Stratiomyidae (Insecta: Diptera): A supplement with revisionary notes and errata. In: Thompson, F. C.; Brake, I. \& Lonsdale, O. eds. Contributions to the Biosystematic Database of World Diptera. Moscow, Myia, vol. III, p.443-484.
Woodley, N. E.; Borkent, A. \& Wheeler, T. A. 2009. Phylogeny of the Diptera. In: Brown, B. V.; Borkent, A.; Cumming, J. M.; Wood, D. M.; Woodley, N. E. \& Zumbado, M. A. eds. Manual of Central American Diptera. Boca Raton, NCR Research Press, p.79-94.

Xerez, R. De \& Pujol-Luz, J. R. 2001. Description of the larva of Vittiger schnusei Kertész, 1909 (Diptera: Stratiomyidae) from Ilha da Marambaia, Rio de Janeiro, Brasil. Studia Dipterologica 8:337-341.

Xerez, R. de; Pujol-LuZ, J. R. \& Viana, G. G. 2002. Descrição da larva de Cosmariomyia argyrosticta Kertész e do pupário de Dactylodeictes lopesi Lindner (Diptera, Stratiomyidae). Revista Brasileira de Zoologia 19(3):747-755.

Xerez, R. DE; Pujol-Luz, J. R. \& Viana, G. G. 2003a. Descrição da larva de Popanomyia femoralis Kertész, 1909 e do pupário de Engicerus major Lindner, 1964 (Diptera, Stratiomyidae). Revista Brasileira de Entomologia 47(3):403-408.

Xerez, R. DE; Pujol-Luz, J. R. \& Viana, G. G. 2003b. Description of the larva of Psephiocera modesta (Lindner, 1949) (Diptera: Stratiomyidae: Pachygastrinae). Studia Dipterologica 10:189-193.

Xerez, R. de; Viana, G. G. \& Pujol-Luz, J. R. 2004. Description of the puparium of Cyphomyia aurifrons Wiedemann and Cyphomyia picta Schiner (Diptera, Stratiomyidae, Clitellariinae) from Ilha da Marambaia, Rio de Janeiro, Brasil. Revista Brasileira de Zoologia 21(1):79-83. 